Национальный медицинский исследовательский центр онкологии

им. Н.Н. Блохина Минздрава РФ (Москва, Россия)

\section{СОВРЕМЕННЫЕ СТРАТЕГИИ}

СТАДИРОВАНИЯ И ЛЕЧЕНИЯ

ГЕПАТОЦЕЛЛЮЛЯРНОГО РАКА

НА ФОНЕ ЦИРРОЗА

В.Ю. Косырев

\section{MODERN STRATEGIES OF STAGING AND TREATMENT OF HEPATOCELLULAR CARCINOMA IN CIRRHOSIS}

В.Ю. Косырев

Доктормедицинских наук, ведущий научный сотрудник, лаборатория интервенщионной радиологии, НИИ клинической и экспериментальной радиологии, НМИЦ онкологии им. Н.Н. Блохина МЗ РФ, 115478, Москва, Каширское шоссе, 23. E-mail:vkosirev@mail.ru.

V.Y. Kosyrev Doctor of Medicine, Leading Researcher, Laboratory of Interventional Radiology, Research Institute of Clinical and Experimental Radiology, N.N. Blokbin National Medical Research Center of Oncology, 115478, Moscow, Kashirskoe shosse, 23. E-mail:vkosirev@mail.ru.

В обзоре приводится эволюция систем стадирования ГЦР по мере обретения новых данных об особенностях его канцерогенеза. Рассматриваются основные системы стадирования: Tumor-Node-Metastasis (TNM), Okuda, Barcelona Clinic Liver Cancer system (BCLC), Cancer of the Liver Italian Program (CLIP), Hong Kong Liver Cancer (HKLC) и другие, с точки зрения их прогностической значимости. Автор приходит к выводу, что многообразие классификаций ГЦР свидетельствует о некоторой неудовлетворенности всех заинтересованных специалистов в существующих системах. Вместе с тем, классификация BCLC, будучи несколько консервативной, несмотря на 20-летнюю историю ее применения, в настоящее время остается одним из наиболее удобных инструментов в определении оптимальной лечебной тактики и прогноза больных ГЦР.

Ключевые слова: гепатоцеллюлярный рак; системы стадирования TNM, $B C L C, H K L C$.

This review presents the evolution of HCC staging systems as the acquisition of new data about the features of its carcinogenesis. The main staging systems are considered: Tumor-Node-Metastasis (TNM), Okuda, Barcelona Clinic Liver Cancer system (BCLC), Cancer of the Liver Italian Program (CLIP), Hong Kong Liver Cancer (HKLC) and others, in terms of their predictive significance. The author comes to the conclusion that the variety of classifications of the HCC indicates a certain dissatisfaction of all interested specialists in existing systems. At the same time, the BCLC classification, being somewhat conservative, despite the 20 -year history of its application, currently remains one of the most convenient tools in determining the optimal therapeutic tactics and prognosis of patients with HCC.

Keywords: hepatocellular cancer; staging systems TNM, BCLC, HKLC. 
$\Gamma$ епатоцеллюлярный рак (ГЦР) печени остается на 6-м месте в мире по частоте выявления в ряду всех злокачественных опухолей и на 4-м месте в мире по причинам смертельных исходов у онкологических больных [1]. Особенностью заболевания, является его тесная связь с хроническими воспалительными процессами в печени, главным образом, имеющими вирусную этиологию, циррозом печени, включая криптогенный, некоторыми обменными нарушениями.

Подтвердив диагноз ГЦР, перед клиницистом возникают следующие задачи, которые требуют максимально быстрого решения - определить прогноз и разработать оптимальную стратегию лечения. Оба эти вопроса напрямую связаны со стадией заболевания. Кроме того, стратификация больных необходима для анализа результатов лечения, обмена достоверной информацией между разными медицинскими центрами, проведения научных исследований.

Очевидно, что хронические, длительно протекающие воспалительные процессы в печени, приводят к функциональному истощению органа и именно это обстоятельство во многих случаях имеет определяющее прогностическое значение, независимо от наличия злокачественной опухоли и степени ее распространенности. Кроме того, недостаточные ресурсы печени зачастую не позволяют провести хирургическое лечение пациентам, даже при минимальном объеме опухолевого поражения. По этой причине, вырабатывая лечебную стратегию у больных ГЦР, сегодня обязательно учитывают помимо, собственно, распространенности опухолевого процесса, функциональное состояние органа. Таким образом, две ключевые задачи при обследовании больных ГЦР заключаются в адекватной оценке объема опухолевого поражения и оценке функционального состояния печени. На первый взгляд, имеющийся сегодня арсенал современных диагностических опций, включая методы инструментальной и лабораторной диагностики, наличие алгоритмов диагностического поиска и различных классификаций, позволяют оперативно и точно получить ответ на все поставленные вопросы. И это суждение справедливо примерно для половины больных ГЦР, в то время, как в отношении другой половины пациентов все оказывается не так очевидно. Это связано с не всегда однозначной интерпретацией данных лучевых методов исследования на фоне цирроза печени, а также с длительным латентным периодом заболевания и отсутствием каких либо проявлений на ранних и специфических клинических проявлений на поздних стадиях опухолевого процесса. Учитывая перечисленные выше обстоятельства, и тот факт, что спектр этиологических факторов цирроза и иных хронических воспалительных состояний печени весьма широк, вопрос создания единой универсальной классификации ГЦР, в которой бы рассматривались все особенности развития заболевания, состояние печени, общее состояние пациента, выделялись и оценивались бы прогностические факторы и популяционные особенности до сих пор остается открытым. Тем не менее, в разное время было разработано более 10 (Tumor-Node-Metastasis (TNM) [2], Okuda[3], Barcelona Clinic Liver Cancer system (BCLC) [4], Cancer of the Liver Italian Program (CLIP) [5], Japan Integrated Staging score (JIS) [6], Hong Kong Liver Cancer (HKLC) [007-12],Chinese University Prognostic Index (CUPI) [8], Group ed'Etudeet de Traitement du Carcinome Hepatocellulaire Prognostic classification (GETCH) [9], Model to Estimate Survival in Ambulatory HCC Patients (MESIAH) [10] и др.) различных классификаций ГЦР. Основные показатели, отражаемые в большинстве этих классификаций - функция печени, уровень АФП, общее состояние пациента (ECOG или Karnofsky), количество и размеры опухолевых узлов, наличие/ отсутствие макро- и микрососудистой инвазии, наличие/ отсутствие внепеченочных проявлений. Эти показатели оказывают существенное влияние на исход заболевания.

Все существующие в настоящее время классификации (системы стадирования) можно условно разделить на прогностические модели (TNM, Okuda, CLIP и др.) и, по сути, алгоритмы с более широкими задачами, определяющие оптимальные пути лечения больных ГЦР (BCLC, HKCL и др.).

Первым важным этапом в систематизации онкологических новообразований, включая ГЦР, стала классификация TNM, применяемая, прежде всего, для описания анатомического распространения опухоли, определяемого её клиническими и гистологическими особенностями. Систематизируя данные об объеме и особенностях опухолевого поражения и, соотнося эту информацию с потенциалом лечебных опций, применимых в данном случае, становится возможным формирование прогностических моделей дальнейшего исхода заболевания. Первая версия классификации TNM вышла в 1968 году. За уже более, чем 50 летний период в онкологии и, в целом в медицине, произошло большое количество событий, повлиявших на эволюцию систем стадирования. Если говорить о ГЦР, то стала очевидной проблема коморбидных состояний печени, играющих важную роль в канцерогенезе и исходе заболевания. Сегодня на принципиально ином уровне находится диагностика рака печени, включая способы лучевой визуализации с применением МРТ с гепатотропными контрастными средами и иммуно-генетические методы исследования опухолевой ткани. Печень остается уникальным органом, ортотопическая трансплантация которого в онкологии, в ряде случаев, может приводить к исцелению, а в большинстве клинических ситуаций позволяет существенно увеличить уровень общей выживаемости. В настоящее время пересмотрена концепция локорегионарного лечения в онкологии, и, применительно к ГЦР, это направление уже рутинно 
применяется на различных этапах развития заболевания, включая самые ранние (при солитарных опухолях менее 2 см) и распространенные процессы, когда объем пораженной паренхимы может превышать $50 \%$. Даже при наличии отдаленных метастазов при ГЦР пациенты сегодня имеют шанс прожить год и более, получая системную терапию. Перечисленные новые возможности в диагностике и лечении ГЦР привели к тому, что удельный вес инкурабельных больных, подлежащих только симптоматическому лечению, сегодня составляет около 10\%, причем, главным образом, невозможность проведения противоопухолевого лечения определяется наличием декомпенсированного цирроза печени.

Все перечисленные обстоятельства, оказали существенное влияние на эволюцию систем стадирования ГЦР.

В основе всех классификаций ГЦР лежат клинические и/или анатомические и/или радиологические признаки. Показатели TNM, в том или ином виде, интегрированы во все современные алгоритмы и прогностические шкалы. Очевидно, что адекватная оценка критерия «Т» (количество, размеры и отношение опухолевых узлов к венам (макрососудистая инвазия)), наряду с показателями «N» и «М» имеют принципиальное значение. В этой связи, важным вопросом является применение максимально достоверных диагностических опций, среди которых в настоящее время, по мнению ряда авторитетных специалистов, наиболее удобным инструментом можно считать систему отчетов и данных визуализации печени LI-RADS (Liver Imaging Reporting and Data System). Она представляет собой стандартизированную классификационную шкалу, оценивающую вероятность наличия у больного гепатоцеллюлярного рака по данным КТ, МРТ, выполненных с контрастным усилением.

Разработанная специалистами в области визуализации печени LI-RADS присваивает наблюдениям категории, которые отражают относительную вероятность опухолевого поражения печени доброкачественной или злокачественной природы, включая ГЦР. Определение категорий базируется главным образом на основных характеристиках изображений, но для повышения достоверности или корректировки категорий LI-RADS используются и вспомогательные признаки, которые могут в большей степени соответствовать злокачественному процессу в целом или ГЦК в частности, тем самым повышая категории до LR-4 либо, напротив, в большей степени свидетельствовать о доброкачественном заболевании, понижая категорию на одну ступень. Если вспомогательные признаки оказываются конфликтующими с основными, то последние имеют приоритет в определении категории $[11,12]$.

Первой системой стадирования опухолей была классификация TNM.
После многолетней предварительной работы в 1968 году Комитет по Номенклатуре и Статистике опухолей (UICC subsequently established a Special Committee on Clinical Stage Classification), созданный Международным противораковым союзом (Union Internationale Contre le Cancer (UICC)) представил первую версию классификации TNM (UICCTNM pocket book, The Livrede Poche, 1st edition). Через 6 лет (в 1974 году) была издана 2-я редакция этой классификации, в которой, в разделе «Печень (включая рак внутрипеченочных желчных протоков)» (С.22.0; С.22.1) критерий Т1 подразумевал наличие любой солитарной опухоли без сосудистой инвазии, не зависимо от размеров узла, а Т2 - наличие солитарной опухоли с сосудистой инвазией, не зависимо от размеров узла или множественные опухолевые узлы, размерами до 5 см [13].

Параллельно, TNM UICC, американским комитетом по раку (American Joint Committee on Cancer, AJCC) разрабатывалась аналогичная классификация, в первой версии которой (издание 1977 года) раздел «Печень» отсутствовал вообще [14, 15], в основу критерия Т были положены количество, размеры и локализация опухолевых узлов в пределах долей печени, независимо от их связи с сосудами. Так Т1 определялся как солитарная опухоль до 3 см, локализующаяся в пределах одной доли, независимо от отношения к сосудам. Т2 подразумевал наличие солитарной опухоли (Т2а) более 3 см в пределах одной доли либо множественных опухолевых узлов любого размера, в пределах одной доли, так же, независимо от взаимоотношения с сосудами. Вместе с тем учитывалось наличие цирроза печени. В зависимости от этого критерия все стадии (I-IV) подразделялись на две: А и В при его отсутствии или наличии, соответственно [15]. Отсутствие показателя инвазии сосудистой стенки существенно снижал достоверность этой системы. Влияние сосудистой инвазии на общую выживаемость сегодня не вызывает сомнения и убедительно продемонстрировано в работе Chen-Hsi H. с соавт. (2015 год), в которой оценивалась общая 3-летняя выживаемость в группе 89 больных ранним ГЦР. Этот показатель в общей группе составил $71 \%$, а в зависимости от наличия/ отсутствия инвазии оказался равным $62,1 \%$ и 92,8\% (p<0,003) [16].

В 1987 году классификации TNM UICC и АJCC были унифицированы и, начиная с 4-й версии, классификация TNM стала единой (UICC/ AJCC). Сегодня эта классификация одобрена всеми национальными Комитетами TNM. В настоящее время в мире актуальна 8-я версия, согласно которой, критерий Т1 разделен на $1 \mathrm{a}$ и $1 б$ для одиночной опухоли без сосудистой инвазии до 2 см и более 2 см, соответственно. Т2 определяется как одиночная опухоль с сосудистой инвазией или множественные опухоли до 5 см [17].

С точки зрения оценки резектабельности при ГцР, эта классификация в целом была и остается, 
подходящей, однако, современная версия не подразумевает адекватного анализа функциональных ресурсов печени.

Первая классификация ГЦР, разработанная Okuda (1985 год), включала долю (процент) опухолевого поражения печени и маркеры функциональной состоятельности печени - асцит, уровень альбумина и билирубина, при этом не учитывала характер внутрии внепеченочного распространения опухоли [3].

Система, разработанная Okuda К. с соавт. более 30 лет назад, явилась первой успешной попыткой объединить анатомические особенности опухоли и степень функциональных изменений печени. При анализе учитывается объем опухолевого поражения (более/ менее $50 \%$ печени) и три показателя тяжести цирроза печени: асцит, уровень сывороточного альбумина и билирубина. Факторами неблагоприятного прогноза считаются: объем поражения паренхимы печени $>50 \%$, уровень альбумина $<3 \mathrm{~g} / \mathrm{dL}$, уровень общего билирубина $>3 \mathrm{mg} / \mathrm{dL}$. При отсутствии любого из этих факторов стадия заболевания определяется как Stage I, a при наличии 1-2 и 3-4 факторов - как Stage II и Stage III, соответственно. Система получила широкое распространение и использовалась на протяжении двух десятилетий. Однако, уязвимым элементом данной системы оставалось то, что ряд важных показателей, таких как инвазия портальной вены, количество и размеры опухолевых узлов, внепеченочное опухолевое поражение не принимались во внимание. Более того, появление новых систем лучевой диагностики, позволяющих выявлять единичные новообразования в печени менее 10 мм, побудили к разработке новой прогностической шкалы, так как грубое определение ранней стадии (Stage I) системы Okuda (поражение менее 50\% объема печени, отсутствие асцита и нормальные показатели альбумина и билирубина), не позволяло адекватно оценивать лечебные перспективы больных ГЦР на начальном этапе развития заболевания [19, 20].

При ретроспективном анализе итальянские исследователи выделили 5 независимых факторов прогноза (класс цирроза Child - Pugh, размер и количество очагов, наличие тромбоза воротной вены (ТВВ) и уровень альфа-фетопротеина (АФП) плазмы) и разработали систему стадирования ГЦР на основании суммы баллов CLIP (Cancer of the Liver Italian Program). Ретроспективные исследования в Канаде, Японии и Европе подтвердили прогностическую значимость CLIP [7], но в крупном анализе ( $\mathrm{n}=906)$, проведенном в Китае, CLIP показала очень слабую корреляцию с выживаемостью [18]. Возможно, подобное расхождение объясняется доминированием в азиатском регионе хронического вирусного гепатита В (ХВГ В), как фактора риска ГЦР, тогда как в европейских странах, Японии и Канаде преобладает хронический вирусный гепатит С (ХВГ С).

Следующим важным шагом в направлении разработки прогностической модели ГЦР стало создание системы CLIP (1998 год), основанной на ретроспективном анализе 435 клинических случаев ГЦР (в подавляющем большинстве - на фоне цирроза печени). Интересным представляется тот факт, что более половины больных $(56,8 \%)$ получали различные виды локорегионарной терапии, а только хирургическое лечение было проведено небольшой группе больных $(2,7 \%)$. Данная система превосходит по прогностической значимости классификации TNM и Okuda, так как учитывает ряд важных параметров: степень выраженности цирроза печени (по Чайлд-Пью), количество опухолевых узлов (солитарный или множественные), внутрипеченочное распространение (> или $<50 \%$ ), уровень АФП (> или $<400 \mathrm{ng} / \mathrm{mL}$ ) и наличие/ отсутствие тромбоза портальной вены (т.е. показатели функционирования печени и объем ее опухолевого поражения). Все эти показатели достаточно легко определяются у больных ГЦР и классификация применима для подавляющего большинства пациентов. В 2000 году были опубликованы данные о валидизации этой системы в ходе проспективного клинического исследования 196 больных ГЦР на фоне цирроза. Prospective validation of the CLIP score: a new prognostic system for patients with cirrhosis and hepatocellular carcinoma. [5]. По этой причине система CLIP в 2002 году была предложена American Hepato-PancreatoBiliary Association и the AJCC в качестве базовой для больных ГЦР [21]. Прогностическая значимость системы была подтверждена для японской, канадской и немецкой когорт больных. Кроме того, в исследовании Zhao W.H. с соавт. (2002 год), которые провели ретроспективный анализ 173 случаев ГЦР (главным образом, на фоне гепатита В) среди китайских больных, перенесших резекцию печени, был продемонстрирован высокий предиктивный потенциал системы. Аналогичные результаты были получены и при изучении выживаемости 131 случая неоперабельных больных корейской популяции после ТАХЭ [22-26].

Однако, несмотря на большую прогностическую значимость CLIP, по сравнению с Okuda, оставалась некоторая неудовлетворенность в отношении анализа ранних стадий ГЦР [27]. По этой причине появились различные модификации. В 2011 году были опубликованы результаты двух работ из одного научного коллектива (The University of Texas M.D. Anderson Cancer Center, Houston) под руководством Kaseb A.O. Были описаны два независимых фактора прогноза ГЦР - эндотелиальный сосудистый фактор роста (VEGF) и инсулино-подобный фактор роста-1 (IGF-1). Было выявлено, что при уровне VEGF в плазме $>450 \mathrm{ng} / \mathrm{ml}$, показатели выживаемости ухудшались. Аналогичным образом, неблагоприятным фактором прогноза выживаемости оказался уровень IGF- $1 \leq 26$ ng/ml. Авторы интегрировали эти факторы в шкалу CLIP и показали, что прогностическая значимость этих модифицированных систем (V-CLIP и I-CLIP, соответственно) существенно выше обычной $[28,29]$. 
Барселонская клиническая классификация рака печени (BCLC) была предложена в 1999 году и до настоящего времени может считаться стандартом прогнозирования и выбора лечения, с учетом периодически вносимых дополнений и изменений. BCLC разработана для больных ГЦР на фоне цирроза печени и представляет из себя алгоритм стратификации пациентов в зависимости от степени выраженности цирроза по классификации Чайлд-Пью (Child-Pugh), общего состояния и распространенности опухолевого поражения (включая количество и размеры опухолевых узлов, наличие/отсутствие сосудистой инвазии). По этим признакам выделяется пять стадий заболевания: от 0 (очень ранняя) до D (терминальная), с наилучшим и максимально неблагоприятным прогнозом, соответственно. Как правило, сложностей в определении этих двух крайних стадий не возникает. Больные на стадиях BCLC-0 (очень ранняя) и BCLC-A (ранняя), в зависимости от объема опухолевого поражения и функциональных ресурсов печени (без выраженной сопутствующей патологии) подлежат наиболее эффективным методам лечения: ортотопическая трансплантация печени, резекция печени, локальная деструкция опухоли. Медиана общей продолжительности жизни этой категории больных превышает 5 лет. Больным с распространенным опухолевым поражением (наличие инвазии воротной вены и/ или отдаленных метастазов) при сохранных/ относительно сохранных ресурсах печени - стадия BCLC-C проводится системное лечение. Показатели общей выживаемости в этой группе больных, как правило, не превышают 12 мес. При декомпенсированном циррозе печени специальное противоопухолевое лечение не проводится и медиана общей выживаемости (на фоне поддерживающей терапии) составляет около 3 мес. Более сложная ситуация с больными промежуточной стадии - BCLC-B, так как объем опухолевого поражения в этой группе может быть весьма вариабельным - от четырех небольших опухолевых узлов до почти полного замещения печени опухолью при условии сохранения функции печени и отсутствия сосудистой инвазии. Стандартным методом лечения больных на промежуточной стадии BCLC является трансартериальная химиоэмболизация. Медиана общей выживаемости больных BCLC-B составляет 20 мес.

Как уже упоминалось, данная система получила широкое распространение и претерпела ряд изменений по мере обретения новых данных клинических исследований.

В версии BCLC 2003 года больным с распространенным опухолевым процессом - стадия BCLC-C (N1M1 + наличие портальной инвазии) рекомендовалось лечение некими «новыми агентами» [30]. В следующей версии (2012 год) той же когорте больных уже рекомендуется конкретный препарат - Сорафениб. А при солитарном опухолевом узле до 2 см в группе больных (BCLC-0), не подлежащих трансплантации рекомендовано выполнять локальную деструкцию опухоли (РЧА/МВА/криоабляцию) [31]. А в модифицированной версии (mBCLC) 2018 года при солитарном опухолевом узле до 2 см в группе больных BCLC-0, независимо от перспектив трансплантации, рекомендуется выполнять локальную деструкцию опухоли или резекцию печени, а больным стадии BCLC-C - системное лечение без указания конкретного препарата, так как к этому моменту уже в России зарегистрированы как минимум два противоопухолевых препарата, а за рубежом их не менее 8 [32].

Система BCLC на долгие годы явилась основным руководством в мире для больных ГЦР на фоне цирроза. Однако, постепенно стала появляться некоторая неудовлетворенность, которая была вызвана разными причинами, и, главным образом, тем, что данный алгоритм существенно ограничивает показания к трансплантации печени. Кроме того, система не учитывает локализацию очагов, не учитывает этиологию цирроза печени, нет четкого представления о противопоказаниях к ТАХЭ и т. д.

В 2010 году группа исследователей из Канады попытались компенсировать некоторые недочеты BCLC, путем ее глубокой модификации. Новый алгоритм получил название Alberta Hepatocellular Carcinoma Algorithm. Основным его отличием было расширение показаний к трансплантации печени, так как в основу отбора пациентов легли не миланские критерии, а показатель - «общий опухолевый объем» (TTV - Total Tumor Volume). Его предиктивное значение, относительно рисков развития рецидивов после ОТТП было продемонстрировано в работе Toso C с соавт. (2008 год). Было установлено, что уровень TTV $\leq 115 \mathrm{~cm}^{3}$ и АФП $\leq 400 \mathrm{ng} / \mathrm{mL}$ являются факторами благоприятного прогноза, а прогностическое значение TTV оказалось существеннее миланских и калифорнийских критериев [33, 34].

Следующим этапом попытки создания универсальной классификации-алгоритма для больных ГцР стала система (Hong Kong Liver Cancer), представленная в 2014 году. Данная система подразумевает более «тонкую" стратификацию больных ( 5 основных стадий и 4 дополнительные), что позволило выделить группы пациентов, которым целесообразнее применять более агрессивные методы лечения. Так, среди больных с ECOG 2-4 и циррозом Чайлд-Пью С (стадия VaHKLC $\backslash$ BCLC-D) выделяется группа больных с небольшими опухолями и без сосудистой инвазии, которые подлежат ОТТП. По мнению авторов HKLC, классификация показала лучший прогностический потенциал, по сравнению с BCLC. Однако, следует отметить, что анализ, положенный в основу HKLC базировался на 3856 клинических случая ГЦР у больных азиатской популяции, с циррозом, преимущественно гепатит-В вирусной этиологии (79,90\%) [35]. Значимость BCLC среди больных этой популяции остается противоречивой [36-38]. 
В мультицентровом исследовании, результаты которого были опубликованы в 2018 году, авторы также выявили большую значимость систем HKLC и MESIAH в популяции больных северной Америки [39].

Вместе с тем, BCLC показала высокую значимость при исследовании европейской популяции больных и при ранних стадиях, поэтому, до настоящего времени, остается важным инструментом стратификации больных ГЦР. В 2016 году научная группа под руководством Бредера В.В. (НМИЦ онкологии им. Н.Н. Блохина) впервые в России провели многофакторный анализ для сравнения классификаций CUPI, CLIP и BCLC. Все классификации подтвердили свою прогностическую значимость. Однако при их прямом сравнении значимых преимуществ какой либо классификации выявлено не было. Автор делает справедливое заключение, что, учитывая одинаковую прогностическую значимость CUPI, CLIP и BCLC, последней следует отдать предпочтение, как наиболее простой в использовании [40].

Таким образом, очевидно, что вопрос создания универсальной классификации ГЦР еще далек от решения. В классификациях отсутствует, как показывает практика, важный фактор прогноза - этиология цирроза. А это в ряде случаев существенно влияет на прогноз. Например, хорошо известно, что мультицентрический рост ГЦР чаще выявляется на фоне хронического цирроза печени у больных HCV [41-43].

В 2017 году был предложен, так называемый, индекс ТОРОНТО, определяющий потенциал развития ГЦР на фоне цирроза. Была показана значимость этиологических факторов цирроза в заболеваемости ГЦР. Наибольшее количество выявленных случаев - 21,7\%, приходилось на когорту больных с предшествующим вирусным гепатитом. В 16,3\% случаев ведущим этиологическим фактором был стеатогепатит, в 4,6\% - аутоиммунное поражение печени. В зависимости от ведущего этиологического фактора цирроза, а также в зависимости от пола, возраста и количества тромбоцитов, все больные разделялись на три группы с низким (<120 баллов), средним (120-240 баллов) и высоким (>240 баллов) риском развития опухоли печени. 5- и 10-летняя кумулятивная заболеваемость ГЦР в этих группах составила: 1,2/2,7\%, 4,4/9,8\% и
15,4/32,1\%, соответственно (данные были получены путем ретроспективного анализа, средний период наблюдения составил 71 месяц). Аналогичные результаты были получены при внешней валидизации, изучив 1144 клинических случая пациентов с циррозом печени (Rotterdam, Netherlands) [44].

Но ресурсы анатомо-функциональных критериев, по-видимому, себя исчерпывают и сегодня исследуется прогностическая значимость молекулярно-генетических факторов, для последующей интеграции их в системы стадирования.

В 2013 году в журнале Gastroenterology была опубликована работа, в которой предлагалась так называемая Шкала 5 генов. Исследователи выявили корреляцию между экспрессией TAF9, RAMP3, HN1, KRT19, RAN и уровнем опухоль-специфической выживаемости и ранних рецидивов после резекции печени [45]. Очевидна необходимость продолжения исследований в этом направлении.

Группа исследователей из Франции, изучая плоидность клеточного ядра обнаружила, что этот показатель существенно увеличивается в опухолях ГЦР, особенно при низкодифференцированных опухолях и при мутации ТР53. По результатам этой работы, было предложено использовать высокую полиплоидность опухолевых клеток как фактор неблагоприятного прогноза [46].

15-20\% ГЦР имеют идентифицируемые и, возможно, таргетируемые молекулярные изменения, но по спектру вызывающих его молекулярных событий этот тип опухолей является крайне гетерогенным [47].

Таким образом, наличие множества классификаций ГЦР отражают сложность проблемы систематизации больных по ведущим этиологическим факторам канцерогенеза, функциональным ресурсам печени, анатомическим особенностям поражения, молекулярно-генетическим признакам в силу высокой гетерогенности больных ГЦР по всем перечисленным критериям. Тем не менее, существующие шкалы и алгоритмы позволяют с определенной степенью достоверности стратифицировать больных, на группы благоприятного и неблагоприятного прогноза, определяя наиболее подходящий метод лечения. Однако, следует учитывать особенности каждой системы стадирования, включая популяционные.

\section{Список литературы}

1. Bray F., Ferlay J., Soerjomataram I.R. et al. Global cancer statistics 2018: GLOBOCAN estimates of incidence and mortality worldwide for 36 cancers in 185 countries // CA: A Cancer Journal for Clinicians. - 2018. - Vol. 68. № 6. - P. 394-424.

2. Lei H.J., Chau G.Y., Lui W.Y., Tsay S.H., King K.L., Loong C.C. et al. Prognostic value and clinical relevance of the 6th Edition 2002 American Joint Committee on Cancer staging system in patients with resectable hepatocellular carcinoma // J Am Coll Surg. - 2006. - Vol. 203. - P. 426-435.

3. Okuda K., Obtsuki T., Obata H., Tomimatsu M., Okazaki N., Hasegawa H. et al. Natural history of hepatocellular carcinoma and prognosis in relation to treatment // Study of 850 patients. Cancer. - 1985. - Vol. 56. - P. 918-928. 
4. Llovet J.M., Bru C., Bruix J. Prognosis of hepatocellular carcinoma: the BCLC staging classification // Semin Liver Dis. - 1999. - Vol. 19. - P. 329-338.

5. [No authors listed] Prospective validation of the CLIP score: a new prognostic system for patients with cirrhosis and hepatocellular carcinoma // The Cancer of the Liver Italian Program (CLIP) investigators. Hepatology. - 2000. Vol. 31. - P. 840-845.

6. Kudo M., Chung H., Osaki Y. Prognostic staging system for hepatocellular carcinoma (CLIP score): its value and limitations, and a proposal for a new staging system, the Japan Integrated Staging Score (JIS score). J Gastroenterol. 2003. - Vol. 38. - P. 207-215.

7. Yau T., Tang V.Y., Yao T.J. et al. Development of Hong Kong Liver Cancer Staging System with treatment stratification for patients with hepatocellular carcinoma // Gastroenterology. - 2014. - Vol. 146. - P. 1691-1700.

8. Leung T.W., Tang A.M., Zee B., Lau W.Y., Lai P.B., Leung K.L. et al. Construction of the Chinese University Prognostic Index for hepatocellular carcinoma and comparison with the TNM staging system, the Okuda staging system, and the Cancer of the Liver Italian Program staging system: a study based on 926 patients // Cancer. - 2002. - Vol. 94. P. $1760-1769$.

9. Chevret S., Trinchet J.C., Mathieu D., Rached A.A., Beaugrand M., Chastang C. A new prognostic classification for predicting survival in patients with hepatocellular carcinoma // Group ed'Etudeet de Traitement du Carcinome Hepatocellulaire. J Hepatol. - 1999. - Vol. 31. - P. 133-141.

10. YangJ.D., Kim W.R., Park K.W. Model to Estimate Survival in Ambulatory Patients With Hepatocellular Carcinoma // Hepatology. - 2012. - Vol. 56. - № 2.

11. Cermy M., Chernyak V., Olivié D. et al. LI-RADS Version 2018 Ancillary Features at MRI // Radiographics. - 2018. Vol. 38. - № 7. - P. 1973-2001.

12. Granata V., Fusco R. Major and ancillary magnetic resonance features of LI-RADS to assess HCC: a novel view and update Infectious Agents // Cancer. - 2017. - Vol. 12. - P. 23.

13. UICC TNM pocket book, «the Livre de Poche» (2nd ed.). - 1974.

14. AJCC Cancer Staging Manual (1st ed.). - 1977.

15. AJCC Cancer Staging Manual (2nd ed.). - 1983.

16. Chen-H H., Chang-Kuo W., Wen-YaoY. et al. Vascular invasion affects survival in early hepatocellular carcinoma // Molecular and clinical oncology. - 2015. - Vol. 3. - P. 252-256.

17. AJCC Cancer Staging Manual (8th ed.). - 2016. Delayed to go into effect 2018.

18. Leung T. et al. Construction of the Chinese University Prognostic Index for hepatocellular carcinoma and comparison with the TNM staging system, the Okuda staging system, and the Cancer of the Liver Italian Program staging system: a study based on 926 patients // Cancer. - 2002. - Vol. 94. - № 6. - P. 1760-1769.

19. Okuda K., Obata H., Nakajima Y., et al. Prognosis of primary hepatocellular carcinoma. Hepatology. - 1984. Vol. 4(Suppl. 1). - P. 3S-6S.

20. Okuda K et al. Natural history of hepatocellular carcinoma and prognosis in relation to treatment study of 850 patients // Cancer. - 1985. - Vol. 56. - № 4. - P. 918-928.

21. Henderson J.M., Sherman M., Tavill A. et al. AHPBA/ AJCC consensus conference on staging of hepatocellular carcinoma: consensus statement // HPB. - 2003. - Vol. 5. - № 4. - P. 243-250.

22. Ueno S., Tanabe G., Sako K. et al. Discrimination value of the new western prognostic system (CLIP score) for hepatocellular carcinoma in 662 Japanese patients // Cancer of the Liver Italian Program. Hepatology. - 2001. Vol. 34. - P. 529-34.

23. Levy I., Sherman M. Liver Cancer Study Group of the University of Toronto // Staging of hepatocellular carcinoma: assessment of the CLIP, Okuda, and Child-Pugh staging systems in a cohort of 257 patients in Toronto. Gut. 2002. - Vol. 50. - P. 881-5.

24. Op den Winkel M., Nagel D., Sappl J. et al. Prognosis of patients with hepatocellular carcinoma // Validation and ranking of established staging-systems in a large western HCC-cohort. PLoS One. - 2012. - P. 7:e45066.

25. Zbao W.H., Ma Z.M., Zbou X.R. et al. Prediction of recurrence and prognosis in patients with hepatocellular carcinoma after resection by use of CLIP score // World J Gastroenterol. - 2002. - Vol. 8. - P. 237-42.

26. Cho Y.K., ChungJ.W., Kim J.K. et al. Comparison of 7 staging systems for patients with hepatocellular carcinoma undergoing transarterial chemoembolization // Cancer. - 2008. - Vol. 112. - P. 352-61.

27. Lin C.Y. et al. Is the Cancer of the Liver Italian Program system anadequate weighting for survival of hepatocellular carcinoma? // Evaluation of intrascore prognostic value among 36 subgroups. Liver Int. - 2009. Vol. 29. - № 1. - P. 74-81.

28. Kaseb A.O., Hassan M.M., Lin E. et al. V-CLIP: Integrating plasma vascular endothelial growth factor into a new scoring system to stratify patients with advanced hepatocellular carcinoma for clinical trials // Cancer. - 2011. Vol. 1. - № 117(11). - P. 2478-88.

29. Kaseb A.O., Abbruzzese J.L., Vauthey J.N. et al. I-CLIP: improved stratification of advanced hepatocellular carcinoma patients by integrating plasma IGF-1 into CLIP score // Oncology. - 2011. - Vol. 80. - № 5-6. - P. 373-81.

30. Llovet M.J., Burroughs A., Bruix J. Hepatocellular carcinoma // Lancet. - 2003. - Vol. 362. - P. 1907-17.

31. Forner A., Llovet M.J., Bruix J. Hepatocellular carcinoma // Lancet. - 2012. - Vol. 379. - P. 1245-55.

32. European Association for the Study of the Liver. EASL Clinical Practice Guidelines: Management of hepatocellular carcinoma // Journal of Hepatology. - 2018. - Vol. 69j. - P. 182-236. 
33. Toso C., Trotter J., Wei A. et al. Total tumor volume predicts risk of recurrence following liver transplantation in patients with hepatocellular carcinoma // Liver Transpl. - 2008. - Vol. 14. - P. 1107-15.

34.Burak K.W., Kneteman N.M. An evidence-based multidisciplinary approach to the management of hepatocellular carcinoma (HCC): The Alberta HCC algorithm // Can J Gastroenterol. - 2010. - Vol. 24 . - № 11. - P. 643-650.

35. Yau T., Tang V.Y., Yao T.J. et al. Development of Hong Kong Liver Cancer Staging System with treatment stratification for patients with hepatocellular carcinoma // Gastroenterology. - 2014. - Vol. 146. - P. 1691-1700.

36. Chan S.L., Mo F.K.F., Johnson P.J. et al. Prospective validation of the Chinese University Prognostic Index and comparison with other staging systems for hepatocellular carcinoma in an Asian population // J Gastroenterol Hepatol. - 2011. - Vol. 26. - P. 340-347.

37. Chen T.W., Chu C.M., Yu J.C. et al. Comparison of clinical staging systems in predicting survival of hepatocellular carcinoma patients receiving major or minor hepatectomy // Eur J Surg Oncol. - 2007. - Vol. 33. - P. 480-487.

38. Cho Y.K., ChungJ.W., Kim J.K. et al. Comparison of 7 staging systems for patients with hepatocellular carcinoma undergoing transarterial chemoembolization // Cancer. - 2008. - Vol. 112. - P. 352-361.

39. Parikh N.D., Scaglione S., Yumeng Li Y. et al. A Comparison of Staging Systems for Hepatocellular Carcinoma in a Multicenter US Cohort // Clinical Gastroenterology and Hepatology. - 2018. - Vol. 16. - P. 781-782.

40. Бредер В.В., Патютко Ю.И., Перегудова М.В., Косырев В.Ю. с соавт. Сравнительный анализ современных систем стадирования гепатоцеллюлярного рака - TNM/AJCC, CUPI, CLIP и BCLC в российской онкологической практике. Опыт Российского онкологического научного центра им. Н.Н. Блохина // Злокачественные опухоли. - 2016. - Т. 18. - № 2.

41. Nakashima O., Kojiro M. Recurrence of hepatocellular carcinoma: multicentric occurrence or intrahepatic metastasis? // A viewpoint in terms of pathology. J Hepatobiliary Pancreat Surg. - 2001. - Vol. 8. - № 5. - P. 404-409.

42. Xиe R., Li R., Guo H. et al. Variable Extent of Intra-tumor Heterogeneity Revealed by Genomic Sequencing of Multiple Lesions in Patients with Hepatocellular Carcinoma // Gastroenterology. - 2016. - Vol. 150. - № 4. P. 998-1008.

43. Wang B., Xia C.Y., Lau W.Y. et al. Determination of clonal origin of recurrent hepatocellular carcinoma for personalized therapy and outcomes evaluation: a new strategy for hepatic surgery. // J Am Coll Surg. - 2013. Vol. 217. - № 6. - P. 1054-1062.

44. Sharma S.A., Kowgier M., Bettina E. et al. Toronto HCC risk index: A validated scoring system to predict 10-year risk of HCC in patients with cirrhosis // Jordan Journal of Hepatology. - 2018. - Vol. 68. - P. 92-99.

45. Nault J.C., De Reyniès A., Villanueva A. et al. A hepatocellular carcinoma 5-gene score associated with survival of patients after liver resection // Gastroenterology. - 2013. - Vol. 145. - P. 176-87.

46. Bou-Nader M., Caruso S., Donne R. et al. Polyploidy spectrum: a new marker in HCC classification // Gut. 2019. - Vol. 0. - P. 1-10.

47. Лазаревич Н.Л., Кривцова О.М., Сковородникова П.А. Молекулярно-генетические особенности клинического прогноза ГЦР // Злокачественные опухоли. - 2016. - № 4, спецвыпуск 1. - С. 40-45.

\section{References}

1. Bray F., Ferlay J., Soerjomataram I.R. et al. Global cancer statistics 2018: GLOBOCAN estimates of incidence and mortality worldwide for 36 cancers in 185 countries. CA: A Cancer Journal for Clinicians. 2018; 68(6): 394-424.

2. Lei H.J., Chau G.Y., Lui W.Y., Tsay S.H., King K.L., Loong C.C. et al. Prognostic value and clinical relevance of the 6th Edition 2002 American Joint Committee on Cancer staging system in patients with resectable hepatocellular carcinoma. J Am Coll Surg. 2006; 203: 426-435. doi: 10.1016/j.jamcollsurg.2006.06.030.

3. Okuda K., Obtsuki T., Obata H., Tomimatsu M., Okazaki N., Hasegawa H. et al. Natural history of hepatocellular carcinoma and prognosis in relation to treatment. Study of 850 patients. Cancer. 1985: 56; 918-928. doi: 10.1002/1097-0142(19850815)56:4<918::aid-cncr2820560437>3.0.co;2-e.

4. Llovet J.M., Bru C., Bruix J. Prognosis of hepatocellular carcinoma: the BCLC staging classification. Semin Liver Dis. 1999; 19: 329-338.

5. [No authors listed] Prospective validation of the CLIP score: a new prognostic system for patients with cirrhosis and hepatocellular carcinoma. The Cancer of the Liver Italian Program (CLIP) investigators. Hepatology. 2000; 31 : 840-845.

6. Kudo M., Chung H., Osaki Y. Prognostic staging system for hepatocellular carcinoma (CLIP score): its value and limitations, and a proposal for a new staging system, the Japan Integrated Staging Score (JIS score). J Gastroenterol. 2003; 38: 207-215.

7.Yau T., Tang V.Y., Yao T.J. et al.Development of Hong Kong Liver Cancer Staging System with treatment stratification for patients with hepatocellular carcinoma. Gastroenterology. 2014; 146: 1691-1700.

8. Leung T.W., Tang A.M., Zee B., Lau W.Y., Lai P.B., Leung K.L. et al. Construction of the Chinese University Prognostic Index for hepatocellular carcinoma and comparison with the TNM staging system, the Okuda staging system, and the Cancer of the Liver Italian Program staging system: a study based on 926 patients. Cancer. 2002; 94: 1760-1769. doi: $10.1002 /$ cncr.10384.

9. Chevret S., Trinchet J.C., Mathieu D., Rached A.A., Beaugrand M., Chastang C. A new prognostic classification for predicting survival in patients with hepatocellular carcinoma. Group ed'Etudeet de Traitement du Carcinome Hepatocellulaire. J Hepatol. 1999; 31: 133-141. 
10. YangJ.D., Kim W.R., Park K.W. Model to Estimate Survival in Ambulatory Patients With Hepatocellular Carcinoma. Hepatology. 2012; 56(2). doi: 10.1002/hep.25680.

11. Cerny M., Chernyak V., Olivié D. et al. LI-RADS Version 2018 Ancillary Features at MRI. Radiographics. 2018; Nov-Dec; 38(7): 1973-2001.

12. Granata V., Fusco R. Major and ancillary magnetic resonance features of LI-RADS to assess HCC: a novel view and update Infectious Agents. Cancer. 2017; 12: 23.

13. UICC TNM pocket book, The Livre de Poche (2nd ed.). 1974.

14. AJCC Cancer Staging Manual (1st ed.). 1977.

15. AJCC Cancer Staging Manual (2nd ed.). 1983.

16. Chen-H H., Chang-Kuo W., Wen-YaoY. et al. Vascular invasion affects survival in early hepatocellular carcinoma. Molecular and clinical oncology. 2015; 3: 252-256.

17. AJCC Cancer Staging Manual (8th ed.). 2016. ISBN 3319406175. Delayed to go into effect 2018.

18. Leung T. et al. Construction of the Chinese University Prognostic Index for hepatocellular carcinoma and comparison with the TNM staging system, the Okuda staging system, and the Cancer of the Liver Italian Program staging system: a study based on 926 patients. Cancer. 2002; 94(6): 1760-1769. doi: 10.1002/cncr.10384.

19. Okuda K., Obata H., Nakajima Y., et al. Prognosis of primary hepatocellular carcinoma. Hepatology. 1984; 4(Suppl. 1): 3S-6S. doi: 10.1002/hep.1840040703.

20. Okuda K. et al. Natural history of hepatocellular carcinoma and prognosis in relation to treatment study of 850 patients. Cancer. 1985; 56(4): 918-928.

21. Henderson J.M., Sherman M., Tavill A. et al. AHPBA/ AJCC consensus conference on staging of hepatocellular carcinoma: consensus statement. HPB. 2003; 5(4): 243-250.

22. Ueno S., Tanabe G., Sako K. et al. Discrimination value of the new western prognostic system (CLIP score) for hepatocellular carcinoma in 662 Japanese patients. Cancer of the Liver Italian Program. Hepatology. 2001; 34: 529-34. doi: 10.1053/jhep.2001.27219.

23. Levy I., Sherman M. Liver Cancer Study Group of the University of Toronto. Staging of hepatocellular carcinoma: assessment of the CLIP, Okuda, and Child-Pugh staging systems in a cohort of 257 patients in Toronto. Gut. 2002; 50: 881-5.

24. Op den Winkel M., Nagel D., SapplJ. et al. Prognosis of patients with hepatocellular carcinoma. Validation and ranking of established staging-systems in a large western HCC-cohort. PLoS One. 2012; 7: e45066.

25. Zhao W.H., Ma Z.M., Zhou X.R. et al. Prediction of recurrence and prognosis in patients with hepatocellular carcinoma after resection by use of CLIP score. World J Gastroenterol. 2002; 8: 237-42.

26. Cho Y.K., ChungJ.W., Kim J.K. et al. Comparison of 7 staging systems for patients with hepatocellular carcinoma undergoing transarterial chemoembolization. Cancer. 2008; 112: 352-61. doi: 10.1002/cncr.23185.

27. Lin C.Y. et al. Is the Cancer of the LiverI talian Program system anadequate weighting for survival of hepatocellular carcinoma? Evaluation of intrascore prognostic value among 36 subgroups. Liver Int. 2009 Jan; 29(1): 74-81.

28. Kaseb A.O., Hassan M.M., Lin E. et al. V-CLIP: Integrating plasma vascular endothelial growth factor into a new scoring system to stratify patients with advanced hepatocellular carcinoma for clinical trials. Cancer. 2011 Jun 1; 117(11): 2478-88. doi: 10.1002/cncr.25791.

29. Kaseb A.O., Abbruzzese J.L., Vauthey J.N. et al. I-CLIP: improved stratification of advanced hepatocellular carcinoma patients by integrating plasma IGF-1 into CLIP score. Oncology. 2011; 80(5-6):373-81.

30. Llovet M.J., Burroughs A., Bruix J. Hepatocellular carcinoma. Lancet. 2003; 362: 1907-17. doi: 10.1016/S01406736(03)14964-1.

31. Forner A., Llovet M.J., Bruix J. Hepatocellular carcinoma. Lancet. 2012; 379: 1245-55.

32. European Association for the Study of the Liver. EASL Clinical Practice Guidelines: Management of hepatocellular carcinoma. Journal of Hepatology. 2018; 69j: 182-236.

33. Toso C., Trotter J., Wei A. et al. Total tumor volume predicts risk of recurrence following liver transplantation in patients with hepatocellular carcinoma. Liver Transpl. 2008; 14: 1107-15. doi: 10.1002/1t.21484.

34. Burak K.W., Kneteman N.M. An evidence-based multidisciplinary approach to the management of hepatocellular carcinoma (HCC): The Alberta HCC algorithm. Can J Gastroenterol. 2010; 24(11): 643-650.

35. Yau T., Tang V.Y., Yao T.J. et al. Development of Hong Kong Liver Cancer Staging System with treatment stratification for patients with hepatocellular carcinoma. Gastroenterology. 2014; 146: 1691-1700. doi: 10.1053/j. gastro.2014.02.032.

36. Chan S.L., Mo F.K.F., Johnson P.J. et al. Prospective validation of the Chinese University Prognostic Index and comparison with other staging systems for hepatocellular carcinoma in an Asian population. J Gastroenterol Hepatol. 2011; 26: 340-347.

37. Chen T.W., Chu C.M., Yu J.C. et al. Comparison of clinical staging systems in predicting survival of hepatocellular carcinoma patients receiving major or minor hepatectomy. Eur J SurgOncol. 2007; 33: 480-487.

38. Cho Y.K., ChungJ.W., Kim J.K. et al. Comparison of 7 staging systems for patients with hepatocellular carcinoma undergoing transarterial chemoembolization. Cancer. 2008; 112: 352-361. doi: 10.1002/cncr.23185.

39. Parikh N.D., Scaglione S., Yumeng Li Y. et al. A Comparison of Staging Systems for Hepatocellular Carcinoma in a Multicenter US Cohort. Clinical Gastroenterology and Hepatology. 2018; 16: 781-782. 
40. Breder V.V., Patyutko Y.I., Peregudova M.V., Kosyrev V.Y. et al. Comparative analysis of modern hepatocellular cancer staging systems - TNM/ AJCC, CUPI, CLIP and BCLC in Russian oncological practice. The experience of the Russian N.N. Blokhin Cancer Research Center. Malignant tumors. 2016; 18(2).

41. Nakashima O., Kojiro M. Recurrence of hepatocellular carcinoma: multicentric occurrence or intrahepatic metastasis? A viewpoint in terms of pathology. J Hepatobiliary Pancreat Surg. 2001; 8(5): 404-409.

42. Xue R., Li R., Guo H. et al. Variable Extent of Intra-tumor Heterogeneity Revealed by Genomic Sequencing of Multiple Lesions in Patients with Hepatocellular Carcinoma. Gastroenterology. 2016; 150(4): 998-1008.

43. Wang B., Xia C.Y., Lau W.Y. et al. Determination of clonal origin of recurrent hepatocellular carcinoma for personalized therapy and outcomes evaluation: a new strategy for hepatic surgery. J Am Coll Surg. 2013; 217(6): 1054-1062.

44. Sharma S.A., Kowgier M., Bettina E. et al. Toronto HCC risk index: A validated scoring system to predict 10-year risk of HCC in patients with cirrhosis. Jordan Journal of Hepatology. 2018; 68: 92-99. doi: 10.1016/j.jhep.2017.07.033.

45. Nault J.C., De Reyniès A., Villanueva A. et al. A hepatocellular carcinoma 5-gene score associated with survival of patients after liver resection. Gastroenterology. 2013; 145: 176-87.

46. Bou-Nader M., Caruso S., Donne R. et al. Polyploidy spectrum: a new marker in HCC classification. Gut. 2019; 0: 1-10. doi: 10.1136/gutjnl-2018-318021.

47. Lazarevich N.L., Krivtsova O.M., Skovorodnikova P.A. Molecular genetic features of the clinical prognosis of HCC. Malignant tumors. 2016; 4(1): 40-45. 